\title{
Focus on Digestive Tract Reconstruction of Laparoscopic Gastrointestinal Tumor Surgery
}

\author{
Song Bin, Su Yanzhuo, Hu Haixia * \\ China-Japan Union Hospital of Jilin University, Changchun, Jilin 130033 \\ abhc311@sina.com
}

Keywords: laparoscopy; gastrectomy; anastomotic operation

\begin{abstract}
Distal gastrectomy, lymph node dissection and digestive tract anastomosis reconstruction under total laparoscopy is the inheritance and development for laparoscope assists in distal gastrectomy. Moreover, it is a hotspot and difficult points in fatal surgery today. This paper makes introduction and discussion on problems such as history, state, key problems and notes of distal gastrectomy digestive tract reconstruction.
\end{abstract}

\section{Introduction}

Compared with general surgical operations, surgical oncology emphasizes at principles of Radical resection and non-tumor technique cooperation. The amplification effect of laparoscope makes cancer lymph node dissection become to be possible for fine anatomy and thorough dissection, its dissection effect is very good due to vision advantage, its radical effect on gastric cancer in early period is increasingly accepted and acknowledged by more and more tumor surgeons. In the past few years, the antecessor of laparoscopic gastric cancer surgery has done plenty and effective work in aspect of clinical practice, they have made detailed illustration and makes it theorization on surgery methods and operation skills in technology level, it also demonstrates safety and feasibility of this surgery, but application of non-tumor technique cooperation by laparoscope are still one of the causes for some tumor surgeons are worried about for this surgical method. This paper will combine with operation steps and technique characteristics for laparoscope assists in radical operation for gastric cancer; it makes discussion on detailed expression and notes of operation principle and technique of non-tumor.

\section{Total laparoscopic gastrointestinal tract Roux-en-Y anastomosis operation technique}

\section{A. General data collection}

In recent years, laparoscopic gastrectomy has obtained more and more successful development in many hospitals in China, its number is obviously increasing and surgery level has been greatly enhanced, as well as gradual accumulation of clinical experience, people begin to pap attention to and explore the hot and difficult problems such as digestive tract reconstruction of laparoscopic gastrectomy. Likewise, digestive tract anastomosis and reconstruction of severe surgical laparoscopy in recent 20 years is worthwhile for Laparoscopic gastrectomy and reconstruction of digestive tract of benign and malignant lesions of stomach to learn reference and development. Laparoscopic gastrectomy digestive tract reconstruction surgery, surgery time and anastomotic leakage rate after surgery are 2 key problems.

Gastrectomy by laparoscope is the difficult point for digestive tract reconstruction, it is also the stitch and knot under laparoscope, it is the causes why surgery time becomes longer. Therefore, there are about $80 \%$ surgeons in America adopting circular stapler or linear cutting closure to complete the anastomose process. They use circular stapler or linear cutting closure to complete the anastomose process, compared with anastomose completed by hand under laparoscope, which kind of method has low stoma leakage, it still needs further research. Higa etc have reported that there were 1000 cases of subtotal gastrectomy and gastrojejunostomy Rou-en-Y anastomosis in 2000, 
the operation time was $60 \mathrm{~min}$, all the patients had none stoma leakage, i ncidence of anastomotic stenosis is $4.9 \%$ (51 examples).

There is Billroth I or II anastomosis of laparoscope by hand, as well as subtotal gastrectomy and gastrojejunostomy $\mathrm{R}$ ou-en-Y anastomosis, it uses circular stapler BillrothIIto complete anastomosis, subtotal gastrectomy and gastrojejunostomy Rou-en-Y anastomosis etc. At present, the usual and representative distal gastrectomy alimentary tract reconstruction by laparoscope is as follows:

(1) BillrothIanastomosis: (1)Delta anastomosis: Kanaya ect invented Delta BillrothIanastomosis under laparoscope by using linear cutting closure, Lee etc made improvement on it. Delta BillrothIanastomosis, it has universal application in clinical application. (2) TST technique anastomosis: TSS technique is the triangulation technique under laparoscope invented by Tanimura in 2008 using linear cutting closure to complete BillrothIanastomosis under laparoscope. (3) BBT technique anastomosis: in 2012, Ikeda etc improved Delta BillrothIanastomosis under laparoscope and invented BBT technique BillrothIanastomosis.

(2) BillrothIIanastomosis: in 1998 and 2008, Kno etc and Lee reported that they used cutting closure under laparoscope to complete subtotal gastrectomy and gastrojejunostomy, they inserted cutting closure gap in stomach and jejunum to complete closure by hand. Kim etc reported that they used cutting closure under laparoscope to complete subtotal gastrectomy and gastrojejunostomy, they inserted cutting closure gap in stomach and jejunum and used cutting closure to close it. In 2012, Du Jianjun etc invented one kind of new linear cutting closure with circular stapler under laparoscope, BillrothIIanastomosis under laparoscope.

(3) Subtotal gastrectomy and gastrojejunostomy Roux-en-Y anastomosis: in 2005, Takaori etc invented distal gastrectomy subtotal gastrectomy and gastrojejunostomy R-oux-en anastomosis under laparoscope. In 2009, Noshiro etc reported the other kind of distal gastrectomy subtotal gastrectomy and gastrojejunostomy R-oux-en anastomosis under laparoscope; it was especially used for patients with small gastric stump after distal gastrectomy.
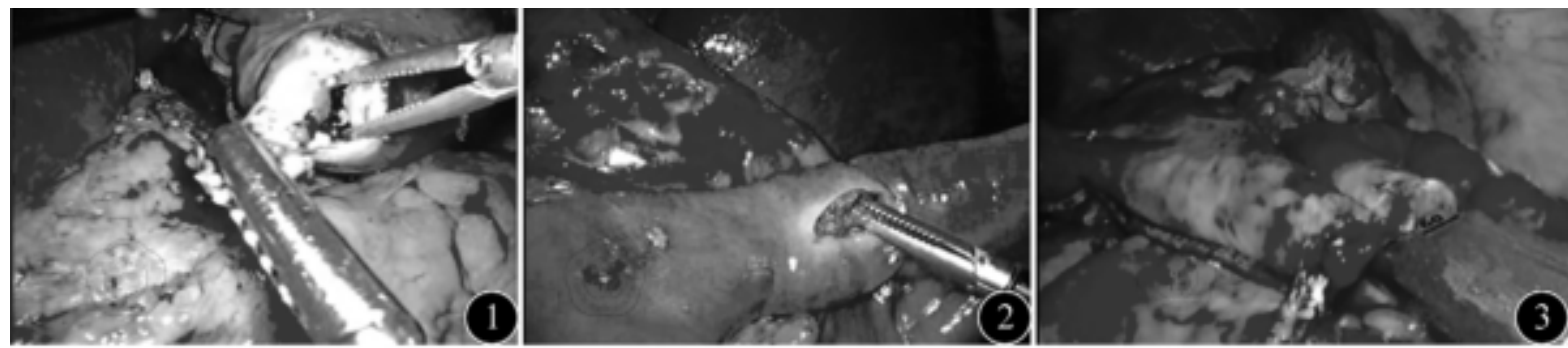

Figure 1 Poke one small hole in greater curvature Figure 2 Poke one small hole in contralateral mesentery Figure 3 side - to - side anastomosis of the large intestine of the stomach
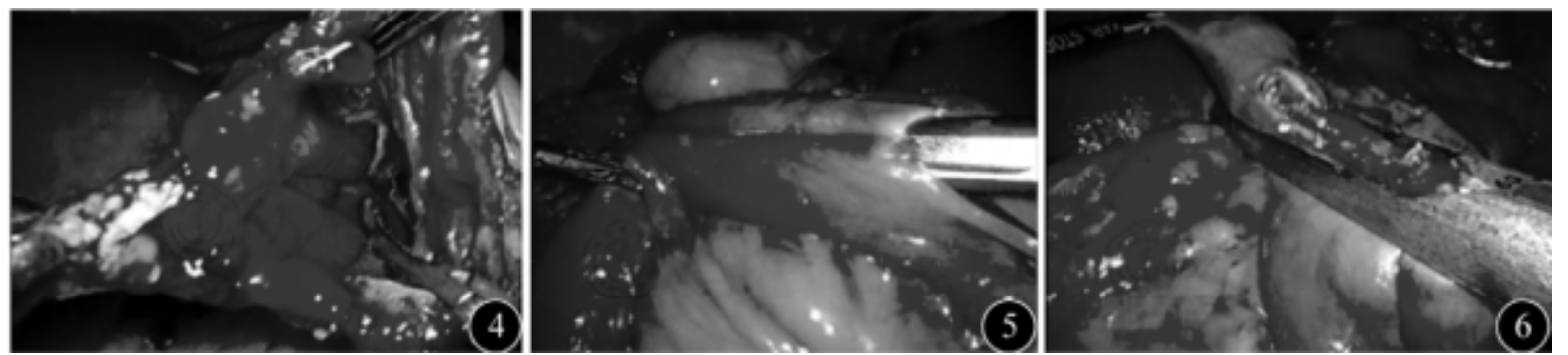

Figure 4 Observe stoma Figure 5 Y side anastomosis of jejunum Figure 6 Cut off and close jejunum and close poke hole 

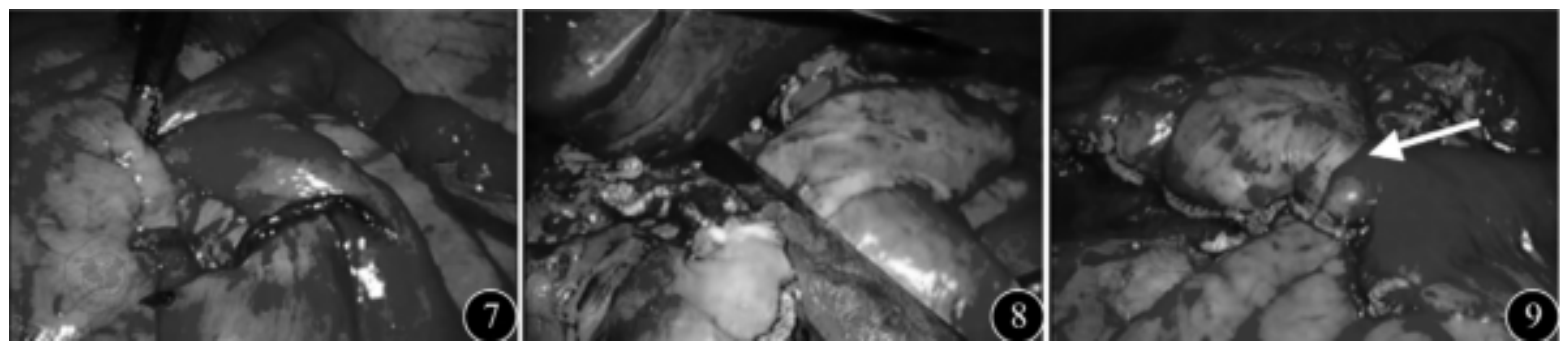

Figure 7 Complete jejunum anastomosis Figure 8 Close uniform opening of stomach and intestine Figure 9 Complete great curvature jejunostomy
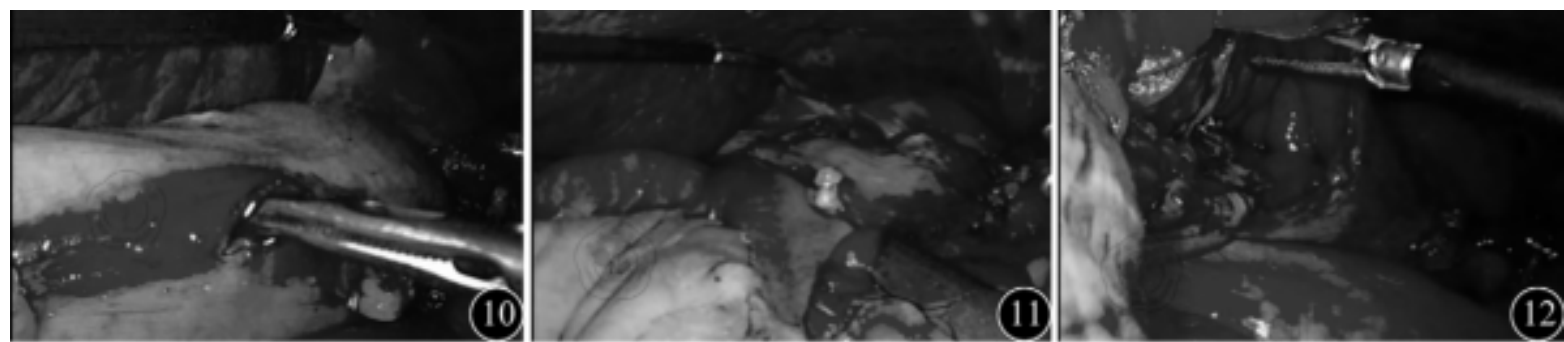

Figure 10 Poke one small hole on $6 \mathrm{~cm}$ away from great curvature of stomach Figure 11 anastomosis diagram of great curvature of stomach jejunum side Figure 12 Observe stoma
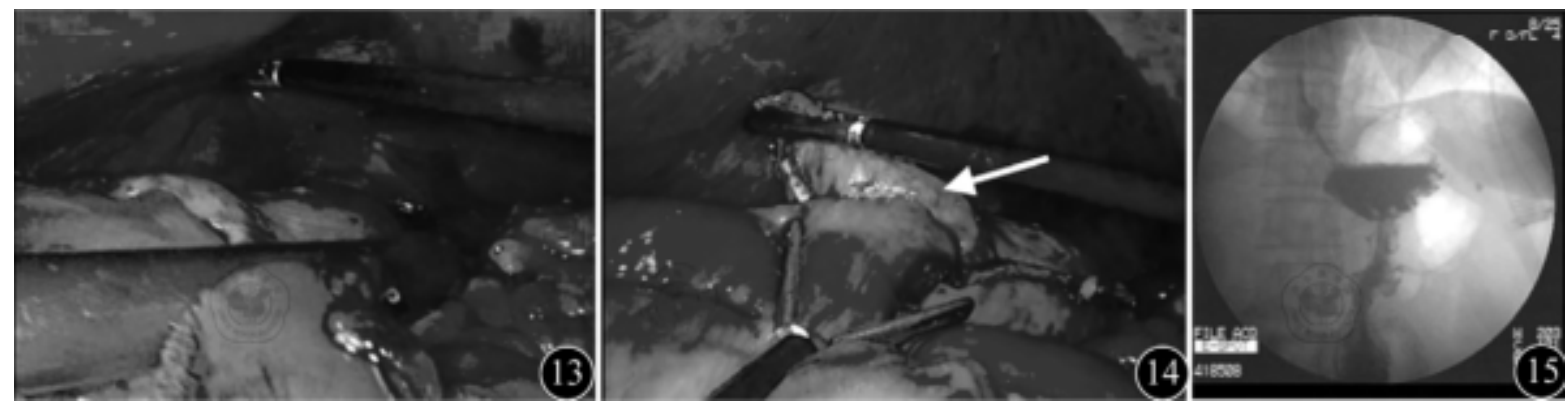

Figure 13 Close uniform opening of stomach and intestine Figure 14 Complete great curvature of stomach and jejunum anastomosis Figure 15 Radiography after surgery

According to peristaltic direction of stomach and intestine, distal resection of stomach under laparoscope Roux-en-Y reconstruction can be divided into converse peristalsis and inverse peristalsis anastomoses methods. Position of Trocar adopts five-hole method, patients lie on their back with head high and foot low, take one longitudinal incision with length of about $1.0 \mathrm{~cm}$ above the umbilicus, pricking pneumoperitoneum and establish $\mathrm{CO}_{2}$ pneumoperitoneum, maintain abdominal pressure at $13 \mathrm{mmHg}$. Place stamp card of $10 \mathrm{~mm}$ above the umbilicus used to observe abdominal cavity condition, respectively place $5 \mathrm{~mm}$ Trocar on $2 \mathrm{~cm}$ of subcostal midline of clavicle and outer umbilical cord level of the rectus abdominis, place $10 \mathrm{~mm}$ Trocar on left subcostal midline of clavicle, place $13 \mathrm{~mm}$ Trocar outer umbilical cord of the rectus abdominis level. Change Trocar of subcostal of the right middle line of the clavicle into $13 \mathrm{~mm}$ Trocar peristaltic gastrointestinal tract Roux-en-Y anastomose.

Anastalsi stomach and intestine Roux-en-Yanastomose: use laparoscope to detect abdominal cavity, implement gastric free and lymph node dissection, and use linear cutting closure to cut off duodenum and stomach, cut off far-end of stomach. Stop pneumoperitoneum, take median incision of about 4 to $6 \mathrm{~cm}$ to cut through abdominal cavity and take out sample, abdominal incision is suitable for taking out sample. Sew up abdominal closure layer by layer, reconstruct pneumoperitoneum. Use ultrasound knife separate mesenterium about 6 to $8 \mathrm{~cm}$ along distal intestinal wall. Bring forth distal jejunum colon to left upper quadrant, respectively one small hole on greater curvature and contralateral mesenter, place straight cut stapler (blue nail of 60mm) great stomach curvature and jejunum (mesangial contralateral) anastomose (figure 1 to figure 3), temporarily place and observe gastroenteric anastomosis whether has active hemorrhage or not(figure 4). Respectively poke one small hole on distal jejunum and proximal jejunum of $40 \mathrm{~cm}$ 
place straight cut stapler(blue nail of $60 \mathrm{~mm}$ ) great stomach curvature and jejunum (ree mesangial proximal side), place linear cutting closure (white nails of $60 \mathrm{~mm}$ ) Jejunum $Y$ side anastomose(anastalsis, figure 5), use linear cutting closure to close uniform opening place of stomach and intestine after confirming whether it has active hemorrhage or not and use linear cutting closure(white nail of 60mm) to cut off, close proximal jejunum and close the uniform opening place(figure 6 and figure 7), complete small intestinal lateral anastomosis. Confirm whether subtotal gastrectomy and gastrojejunostomy has active hemorrhage or not, and use linear cutting closure (white nail of 60mm) to close uniform opening place of stomach and intestine (blue nail) after confirming stoma is bigger than $2.5 \mathrm{~cm}$, complete lateral jejunal anastomosis of jejunal side of the great curvature of the vermicularis (figure 8 and figure 9). Induce duodenal stump to drainage tube on right upper quadrant.

Peristaltic gastrointestinal tract Roux-en-Yanastomosis: except for Roux-en-Y, the other steps coincide with converse gastrointestinal tract Roux-en-Y. Respectively poke one small hole on greater curvature $6 \mathrm{~cm}$ away and contralateral mesentery (free mesangial distal side) and place (distal gastric direction) linear cutting closure (blue nail of $60 \mathrm{~mm}$ ) great curvature of stomach and jejunum (mesangial contralateral) anastomose (figure 10 and figure 11). Confirm gastroenteric anastomosis has no active hemorrhage (figure 12), use linear cutting closure (blue nail of 60mm) after confirming stoma is bigger than $2.5 \mathrm{~cm}$, close uniform opening place of stomach and intestine, complete lateral anastomosis of jejunum with peristalsis and great curvature of stomach (figure 13 and figure 14).

\section{Treatment after surgery of digestive tract reconstruction in gastrointestinal tumors surgery under laparoscope}

It should give decompression, fluid resuscitation, acid suppression, symptomatic treatment to stomach and intestine, use ioversol (with iodine content of $320 \mathrm{mg} / \mathrm{ml}$ ) to make radiography (figure15) after surgery of 3 to 5 days, it should confirm there is no anastomotic leak and extract gastrointestinal decompression tube, give patients clear stream food, and gradually evolves it to stream food and half stream food. The notes during surgery: 1 . when dealing with mesostenium, we should cut small section of small intestine along intestinal wall, which can avoid damaging autonomic nerve of small intestine; 2 . choose lateral deviated point of greater curvature, which can avoid gastrointestinal anastomosis line overlapping with hole closing line; 3. it dose not close after gastroenteroanastomosis and open together, make small intestine anastomosis, which is favorable to observe whether gastrointestinal anastomosis has active hemorrhage or not; 4 . amputation and closure of the small intestine should be treated together, drag the front wall of small intestine toward direction of mesenteric contralateral and make coincidence line of small intestine stagger, which can avoid straitness of small intestine hole, and it can avoid astrointestinal anastomosis line overlapping with hole closing line; 5 . it is difficult to observe posterior wall of anastomosis when closure of small intestinal rupture and perforation, we should pay attention to confirm incomplete closure; 6 . there may be some leakage of gastric juice and bile on operation, use normal saline to completely wash it after anastomosis, it should avoid intra-abdominal abscess.

The patients in the whole group have smoothly completed radical operation for gastric cancer, their recovery is very good after surgery, and they have no severe complication during staying in hospital, they can have normal dinner and sleep after leaving hospital, their dinner amount is from one third to half of that before surgery. We have made random visit over 6 months on patient, we miss none and there is no tumour recidivation, all the patients have completed data collection in the random visit period. BMI, blood glucose and insulin dosage comparison of patients after surgery, compared with that before surgery, every kind of index of patients in radical operation for carcinoma of stomach after digestive tract reconstruction has obviously changes $(\mathrm{P}<0.05)$, and Billroth II or Roux-en-Y has obvious changes than that of Billroth I group $(P<0.05)$, please see figure 16, 17 and 18. 

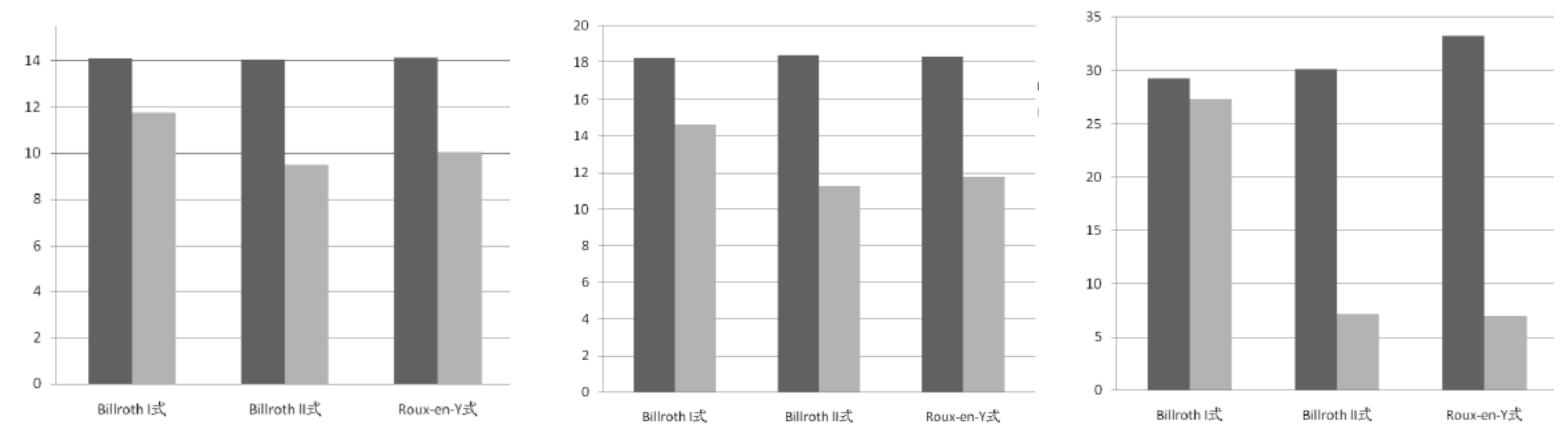

Figure 16 Fasting blood glucose change comparison before and after surgery Figure 17 Blood glucose change comparison after dinner of $2 \mathrm{H}$ before and after surgery Figure 18 Insulin dosage change comparison before and after surgery

\section{Experience on distal gastrectomy digestive tract reconstruction by total laparoscopy}

In recent years, laparoscopic gastrectomy has obtained more and more successful development in many hospitals in China, its number is obviously increasing and surgery level has been greatly enhanced, as well as gradual accumulation of clinical experience, people begin to pap attention to and explore the hot and difficult problems such as digestive tract reconstruction of laparoscopic gastrectomy. Likewise, digestive tract anastomosis and reconstruction of severe surgical laparoscopy in recent 20 years is worthwhile for Laparoscopic gastrectomy and reconstruction of digestive tract of benign and malignant lesions of stomach to learn reference and development. Laparoscopic gastrectomy digestive tract reconstruction surgery, surgery time and anastomotic leakage rate after surgery are 2 key problems.

Cultivating control and ruling ability of seaming and inserting needle, choosing needle and suture line with proper size and length, expertly mastering knotting technique are the important base for digestive tract reconstruction under laparoscope. Choosing proper laparoscope and using cutting closure or circular stapler is the important guarantee for digestive tract reconstruction. In order to avoid tissue ischemia or anastomotic distortion, proper position of cutting closure or circular stapler as well as check whether binding edge has haemorrhage or not after cutting closure or circular stapler hits kiss are 2 key problems for digestive tract reconstruction surgery by laparoscope. Choosing relatively stable surgery, doctors as well as relatively stable nurses on and below surgey platform are the important guarantee for anastomosis and reconstruction under laparoscope.

Preparing for complete and diversified relative surgery appliances and materials are the important part for the successful surgery for anastomosis and reconstruction under total laparoscopic gastrectomy. Especially, we should prepare for laparoscopic liver retractor, laparoscopic needle holder with radian in head, which is very important for the smooth distal gastrectomy alimentary tract reconstruction under total laparoscopy. With the enhancement in technique level and operation level, digestive tract anastomosis becomes increasingly popular with people. In order to effectively solve this problem, scholars both in China and abroad are making research on the ideal anastomosis method. We firstly apply linear cutting closure in China and complete Roux-en-Y subtotal gastrectomy and gastrojejunostomy under total laparoscopy to demonstrate its safety and feasibility. In a word, research on digestive tract reconstruction after distal gastrectomy is very little, especially that prospective RCT result is very little, which lacks of evidence-based medical evidence with enough grade, now there is no regulation to follow acknowledged by people. It is believed that with the deepening of research, we can display the advantages of bulk cases of every center, integrate resources and get powerful evidence-based medical evidence.

\section{References}

[1] Guidelines for Rational Use of Drugs for Malignant Gastrointestinal Tumors [J]. China Rational Drugs Use Exploration, 2017, 14(09):5-54. 
[2] Jing Huazhong, Liu Hongbin. Application State of Laparoscope in Advanced Gastric Carcinoma [J]. Chinese Journal of Minimally Invasive Surgery, 2014, 14(07):662-663+672.

[3] Du Jianjun. Operation and Practice of Distal Gastrectomy Digestive Tract Reconstruction by Total Laparoscopy [J]. Chinese Journal of General Surgical Surgery (electronic version), 2014, 8(04):313-319.

[4] Liang Han. Principles and Notes of Standardized Surgery for Plasmacytoma between Stomach and Intestine [J]. Chinese Journal of Practical Surgery, 2015, 35(04):391-394.

[5] Liao Wenqiang, Ge Haiyan. Application Progress of Fast Track Surgery [J]. Surgery Research and New Technology, 2016, 5(02):118-121. 